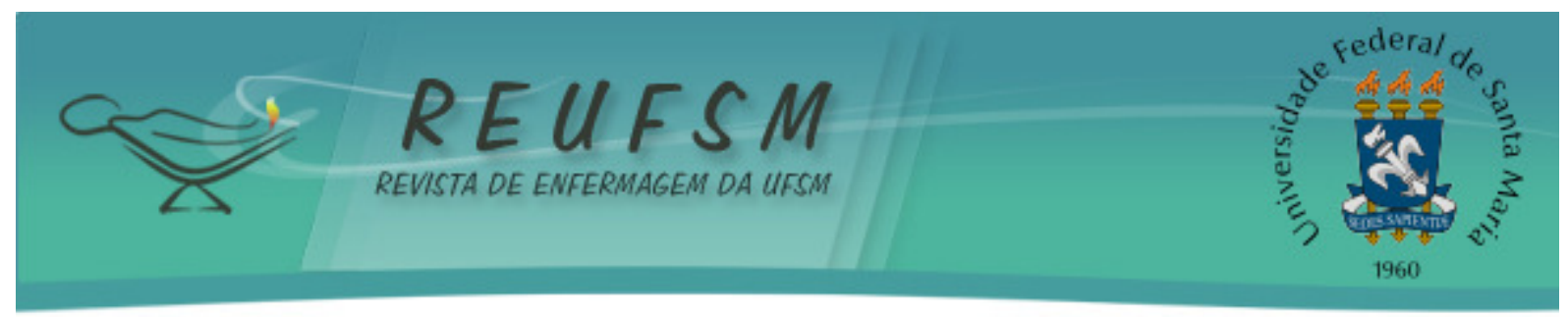

ARTIGO ORIGINAL

\title{
CARACTERIZAÇÃO DOS CLIENTES EM TRATAMENTO RADIOTERÁPICO EM UM SERVIÇO NO SUL DO BRASIL*
}

\section{CHARACTERIZATION OF THE CLIENTS IN RADIOTHERAPIC TREATMENT IN A SERVICE IN SOUTHERN BRAZIL}

\section{CARACTERIZACIÓN DE LOS CLIENTES EN TRATAMIENTO RADIOTERÁPICO EN UN SERVICIO EN EL SUR DE BRASIL}

\author{
Juliana Graciela Vestena Zillmer ${ }^{1}$ \\ Lílian Moura de Lima² \\ Aline Machado Feijó ${ }^{3}$ \\ Eda Schwartz ${ }^{4}$ \\ Claudia das Neves Hisse ${ }^{5}$ \\ Aline da Costa Viegas ${ }^{6}$ \\ Bianca Pozza dos Santos
}

RESUMO: Objetivo: caracterizar os clientes com câncer em tratamento radioterápico em um Centro Regional de Oncologia da Região Sul do Brasil. Método: estudo quantitativo descritivo. Fizeram parte do estudo 147 clientes com câncer em tratamento radioterápico no período de Março de 2006 a Julho de 2008. A coleta dos dados ocorreu por meio de um questionário semiestruturado. Para a análise utilizou-se a estatística básica, por meio do software Epi Info (6.04). Resultados: houve predomínio de pacientes do sexo masculino, cor branca, estado civil casado/com companheiro, faixa etária acima dos 60 anos. A procedência foi predominantemente da área urbana, com renda de um a três salários mínimos, ensino fundamental incompleto. Os cânceres de nasofaringe foram os de maior frequência e o tratamento que antecedeu a radioterapia foi a cirurgia. Conclusão: a caracterização dos clientes permite o desenvolvimento de estratégias direcionadas à qualificação da atenção a saúde a essa população.

Descritores: Enfermagem; Oncologia; Radioterapia; Perfil de saúde; Estudos epidemiológicos.

\footnotetext{
*Pesquisa financiada pela Fundação de Amparo à Pesquisa do Estado do Rio Grande do Sul (FAPERGS), sob o $n^{\circ}$ 05/2279.2 PROADE 3.

${ }^{1}$ Enfermeira. Mestre em Enfermagem. Doutoranda pelo Programa de Pós-Graduação em Enfermagem da Universidade Federal de Santa Catarina. Docente da Faculdade de Enfermagem da UFPel. Membro do Núcleo de Condições Crônicas e suas Interfaces (NUCCRIN) e do Núcleo de Estudos e Assistência em Enfermagem e Saúde às Pessoas com Doenças Crônicas (NUCRON). Pelotas, RS, Brasil. E-mail: juzillmer@gmail.com

${ }^{2}$ Enfermeira. Mestre em Enfermagem. Doutoranda pelo Programa de Pós-Graduação em Enfermagem da UFPel. Membro do NUCCRIN. Pelotas, RS, Brasil. E-mail: lima.lilian@gmail.com

${ }^{3}$ Mestre em Enfermagem. Doutoranda pelo Programa de Pós-Graduação em Enfermagem da UFPel. Enfermeira do Hemocentro Regional de Pelotas (HEMOPEL). Membro do NUCCRIN e do Núcleo de Pesquisa em Saúde Rural e Sustentabilidade. Pelotas, RS, Brasil. E-mail: aline_feijo@yahoo.com.br

${ }^{4}$ Enfermeira. Pós-Doutora em Enfermagem. Docente da Faculdade de Enfermagem e do Programa de Pós-Graduação em Enfermagem da UFPel. Pesquisadora do NUCCRIN e vice-líder Núcleo de Pesquisa em Saúde Rural e Sustentabilidade. Pelotas, RS, Brasil. E-mail: eschwartz@terra.com.br

${ }^{5}$ Enfermeira. Mestre em Enfermagem. Membro do NUCCRIN. Pelotas, RS, Brasil. E-mail: claudiah.08@hotmail.com ${ }^{6}$ Enfermeira. Mestranda pelo Programa Pós-Graduação em Enfermagem da Faculdade de Enfermagem da UFPel. Bolsista de Demanda Social (CAPES). Membro do NUCCRIN e do Núcleo de Pesquisa em Saúde Rural e Sustentabilidade. Pelotas RS, Brasil. E-mail: alinecviegas@hotmail.com

${ }^{7}$ Enfermeira. Mestranda pelo Programa Pós-Graduação em Enfermagem da Faculdade de Enfermagem da UFPel. Bolsista de Demanda Social (CAPES). Membro do NUCCRIN e do Núcleo de Pesquisa em Saúde Rural e Sustentabilidade. Pelotas, RS, Brasil. Brasil. E-mail: bi.santos@bol.com.br
} 


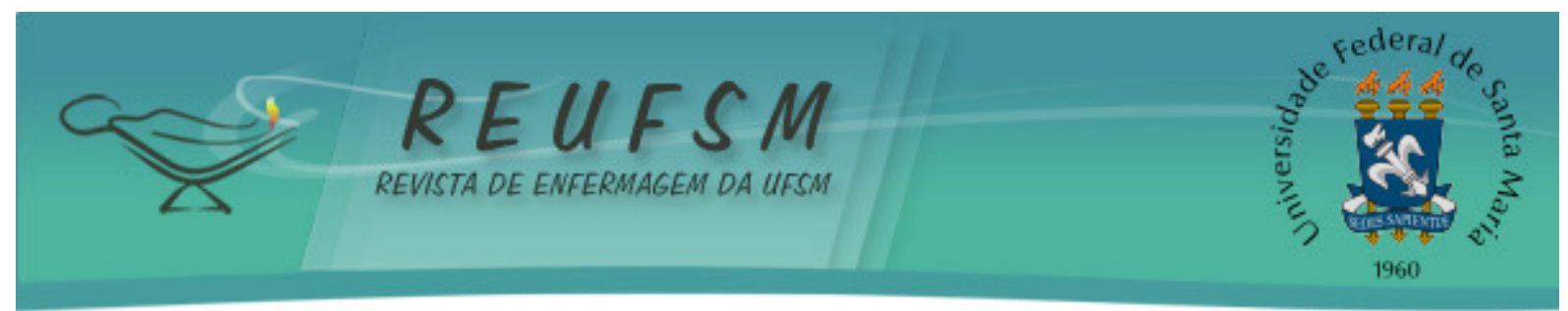

ABSTRACT: Aim: to describe the characteristics of clients with cancer undergoing radiotherapy in a Regional Center of Oncology in Southern Brazil. Method: it is a descriptive quantitative study. Study participants were 147 clients with cancer undergoing radiotherapy from March 2006 to July 2008. Data collection occurred through a semistructured questionnaire. It was used the basic statistics for the analysis using the software Epi Info (6:04). Results: there was predominance of male patients, white color, marital status married/with partner, age over 60 years. The origin was predominantly from the urban area, with income from one to three minimum wages, incomplete primary education. Cancers of the nasopharynx were the most frequent and the treatment prior to radiotherapy was the surgery. Conclusion: the characterization of the clients allows the development of targeted strategies on qualified health care to this population.

Descriptors: Nursing; Medical oncology; Radiotherapy; Health profile; Epidemiologic studies.

RESUMEN: Objetivo: caracterizar los clientes con cáncer en tratamiento radioterápico en un Centro Regional de Oncología de la Región Sur de Brasil. Método: estudio cualitativo, descriptivo. Fueron estudiados 147 clientes con cáncer en tratamiento radioterápico en el periodo de Marzo de 2006 a Julio de 2008. La colecta de los datos ocurrió por medio de un cuestionario semiestructurado. Para el análisis se utilizó la estadística básica, a través del software Epi Info (6.04). Resultados: hubo predominio de pacientes del sexo masculino, color blanco, casados/con compañero, edad superior a 60 años. La procedencia fue predominantemente del área urbana, con renta de un a tres salarios mínimos, enseñanza fundamental incompleta. Los cánceres de nasofaringe fueron los más incidentes y el tratamiento que antecedió la radioterapia fue la cirugía. Conclusión: la caracterización de los clientes permite el desarrollo de estrategias direccionadas a la calificación de la atención a la salud de esa población.

Descriptores: Enfermería; Oncología médica; Radioterapia; Perfil de salud; Estudios epidemiológicos.

\section{INTRODUÇÃO}

As mudanças ocorridas no perfil demográfico e epidemiológico do país proporcionaram elevação na ocorrência das doenças crônicas não transmissíveis, dentre as quais está o câncer. Esse é considerado uma doença crônica complexa, que compromete significativamente a vida dos indivíduos no âmbito biológico, social e afetivo, causando transformações na vida desses sujeitos, e por consequência, exigindo uma assistência à saúde qualificada. O câncer é evidenciado como uma enfermidade biológica, sendo um importante problema social, figurando entre as principais causas de mortalidade no Brasil e no mundo. ${ }^{1}$

No cenário brasileiro, as estimativas para o ano 2012 e 2013 assinalam a ocorrência de 518.510 casos novos de câncer, sendo os tipos mais incidentes os cânceres de pele não melanoma, de próstata e de pulmão no sexo masculino e os cânceres de pele não melanoma, da mama e do colo do útero no sexo feminino. Quando se discute os fatores de risco para essa enfermidade, destacam-se os fatores genéticos, agentes externos, como poluição do ar e exposição excessiva ao sol, hábitos comportamentais, como tabagismo e alcoolismo, entre outros. ${ }^{1}$

Para o tratamento do câncer, estão disponíveis diferentes modalidades terapêuticas, sendo uma delas a radioterapia, uma forma de tratamento local e/ou regional, indicada de forma exclusiva ou associada a outras opções de tratamento, como a quimioterapia e a cirurgia, e com objetivo de cura, remissão, profilaxia ou terapêutica paliativa. ${ }^{2}$

Nos últimos anos, ocorreu um aumento expressivo no número de pacientes oncológicos atendidos pelas Unidades de Alta Complexidade do Sistema Único de Saúde (SUS), refletindo uma melhora na capacidade do sistema em aumentar o acesso aos recursos de tratamento especializado. ${ }^{1}$ 


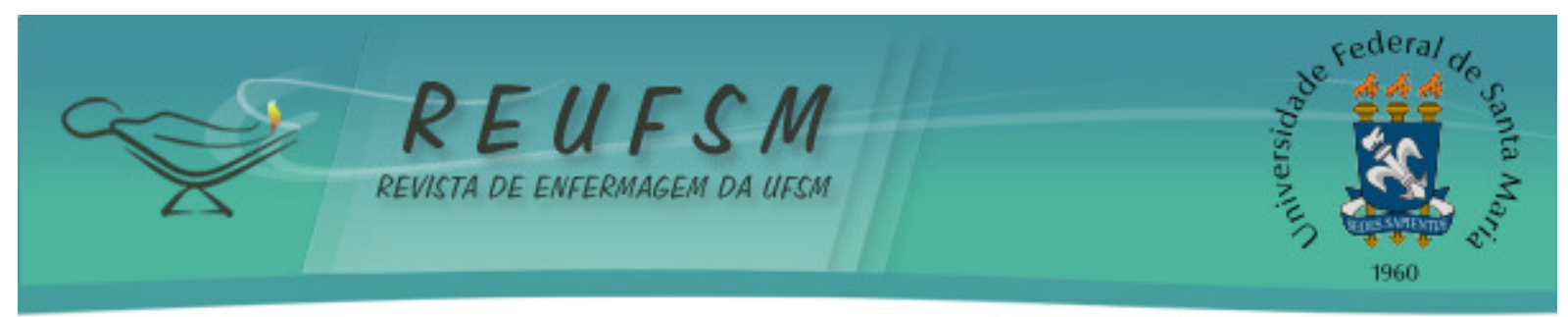

A assistência na área oncológica é uma das mais onerosas para os cofres públicos, abrangendo desde custos diretos com a prevenção, diagnósticos e tratamentos até custos indiretos relacionados à incapacidade produtiva do doente, da morbidade, do tratamento e a própria mortalidade. ${ }^{1}$

No Brasil, o Ministério da Saúde garante a assistência ao paciente oncológico, por meio de políticas públicas e leis, as quais regulamentam a atenção destinada a essa parcela da população. Dentre essas, cita-se a Lei $n^{\circ} 12.732$ de 22 de novembro de 2012, estabelecendo que o paciente com câncer tenha o direito de se submeter gratuitamente ao tratamento oncológico pelo SUS, sendo estimado um prazo de até 60 dias a partir da confirmação do diagnóstico da doença, ou ainda iniciá-lo em prazo menor, de acordo com a sua necessidade. ${ }^{3}$

Assim, conhecer o perfil epidemiológico do câncer em determinada região é relevante, uma vez que a partir das informações pode haver a implementação de políticas públicas e de programas à melhoria da atenção as pessoas assistidas pelo SUS. Também pode contribuir com a produção de estudos que traçam o perfil epidemiológico do câncer nos municípios brasileiros, que ainda são escassos. ${ }^{4}$

Em decorrência do aumento da prevalência e da incidência de câncer na população em geral, é importante compreender as características dos clientes com câncer que frequentam os serviços de saúde. Não menos importante é conhecer a assistência prestada a essa população. ${ }^{5}$

Frente aos desafios de atuar junto ao cliente oncológico em tratamento, sobretudo o radioterápico, de modo a qualificar a assistência de enfermagem, surgiu à necessidade de investigar esta demanda. Entende-se que conhecer as características desses indivíduos é uma prática que se faz necessária, pois fornecerá subsídios para desenvolver estratégias de fortalecimento de ações em saúde específicas. Neste contexto, propôs-se a seguinte questão norteadora “Qual o perfil dos clientes com câncer em tratamento radioterápico?”.

Portanto, o presente trabalho teve como objetivo caracterizar os clientes com câncer em tratamento radioterápico em um Centro Regional de Oncologia da Região Sul do Brasil.

\section{MÉTODO}

Este estudo trata de uma investigação quantitativa descritiva, realizada no Centro Regional de Oncologia. Esse centro recebe pessoas de ambos os sexos que estão em tratamento para algum tipo de câncer, e realiza o atendimento médio de 20 pacientes a cada dia, com 250 novos usuários a cada ano.

Assim, a população foi constituída por uma amostra de conveniência, resultando em 147 clientes com câncer em tratamento radioterápico no Centro Regional de Oncologia que frequentaram o serviço no período de Março de 2006 a Julho de 2008. Os critérios de inclusão foram: idade maior ou igual a 18 anos, estar ciente do diagnóstico de câncer e estar em tratamento no Centro Regional de Oncologia. Aqueles clientes que se enquadraram nos critérios de inclusão foram convidados a participar da pesquisa mediante assinatura do Termo de Consentimento Livre e Esclarecido.

$O$ instrumento contendo questões sociodemográficas, econômicas, relacionadas ao tipo de câncer e tratamento foi aplicado e, posteriormente codificado, por acadêmicos que receberam uma capacitação prévia do pesquisador responsável. Em seguida, os dados foram digitados no software Epi Info (versão 6.04), procedendo-se dupla digitação e pareamento dos bancos para verificar possíveis inconsistências nos dados e, se necessário, realizar as correções.

A análise estatística foi realizada no software Epi Info (versão 6.04). Estudaram-se as variáveis sócio-demográficas: sexo, cor da pele, escolaridade, situação conjugal, procedência, situação na previdência e renda familiar; as variáveis relacionadas aos fatores de risco: tabagismo, tempo de tabagismo, alcoolismo, uso de chimarrão; e as 


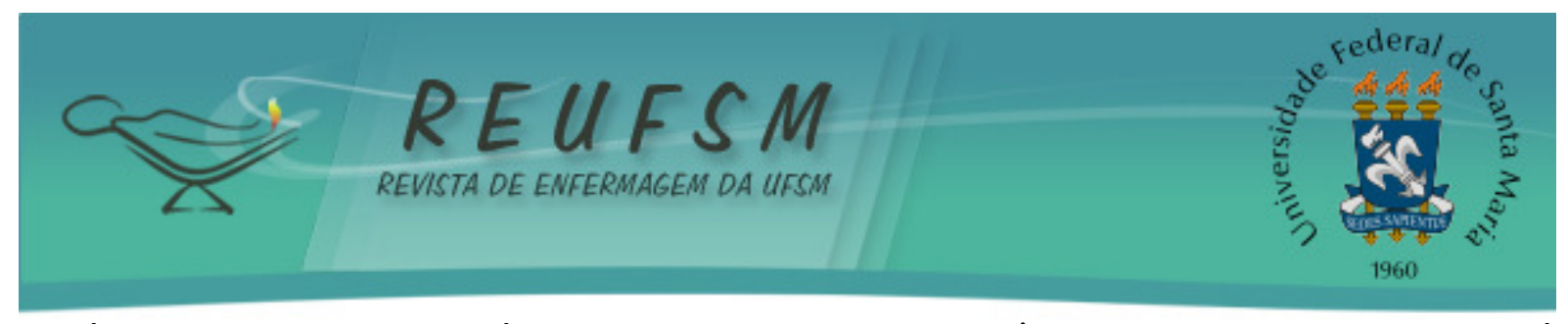

variáveis sobre as características da doença: tipo de câncer e tratamento anterior à radioterapia. Para tanto, foi utilizada a estatística básica com distribuição de frequências.

Foram seguidas as normas para pesquisa envolvendo seres humanos estabelecidas pela Resolução $n^{\circ} 196 / 96$ do Conselho Nacional de Saúde. ${ }^{6}$ O projeto de pesquisa foi aprovado pelo Comitê de Ética e Pesquisa da Faculdade de Medicina da Universidade Federal de Pelotas, sob número 028/06.

\section{RESULTADOS}

Foram entrevistados 147 clientes oncológicos em tratamento radioterápico no Centro Regional de Oncologia. A Tabela 1 apresenta as variáveis sociodemográficas dos clientes oncológicos, com relação ao sexo, faixa etária, cor da pele, escolaridade, situação conjugal, procedência, situação previdenciária e renda familiar. 


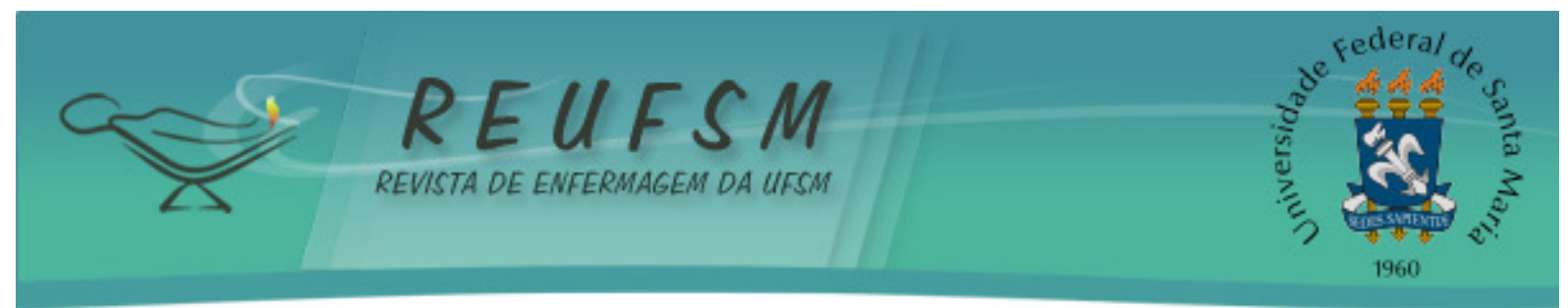

Tabela 1 - Distribuição dos clientes oncológicos em tratamento radioterápico no centro regional de oncologia segundo as características sociodemográficas e econômicas, 2008.

\begin{tabular}{|c|c|c|}
\hline Características sociodemográficas & $\mathrm{N}(147)$ & $\%$ \\
\hline \multicolumn{3}{|l|}{ Sexo } \\
\hline Masculino & 85 & 57,8 \\
\hline $\begin{array}{r}\text { Feminino } \\
\text { Faixa etária }\end{array}$ & 62 & 42,2 \\
\hline $20-39$ anos & 03 & 2,0 \\
\hline $40-59$ anos & 56 & 38,1 \\
\hline 60 anos ou mais & 88 & 58,9 \\
\hline \multicolumn{3}{|l|}{ Cor da pele } \\
\hline Branca & 122 & 83,6 \\
\hline Preta & 14 & 9,6 \\
\hline Parda & 10 & 6,8 \\
\hline \multicolumn{3}{|l|}{ Escolaridade } \\
\hline Não alfabetizado & 24 & 16,3 \\
\hline Ensino fundamental incompleto & 80 & 54,4 \\
\hline Ensino fundamental completo & 19 & 12,9 \\
\hline Ensino médio incompleto & 07 & 4,8 \\
\hline Ensino médio completo & 11 & 7,5 \\
\hline Ensino superior incompleto & 01 & 0,7 \\
\hline Ensino superior completo & 05 & 3,4 \\
\hline \multicolumn{3}{|l|}{ Situação conjugal } \\
\hline Casado/Com companheiro & 86 & 58,5 \\
\hline Solteiro & 15 & 10,2 \\
\hline Viúvo & 32 & 21,8 \\
\hline Separado/Divorciado & 14 & 9,5 \\
\hline \multicolumn{3}{|l|}{ Procedência } \\
\hline Urbana & 106 & 72,1 \\
\hline Rural & 41 & 27,9 \\
\hline \multicolumn{3}{|l|}{ Situação na previdência } \\
\hline Pensionista & 16 & 11,3 \\
\hline Aposentado & 73 & 51,4 \\
\hline Auxílio doença & 28 & 19,7 \\
\hline Nenhuma & 25 & 17,6 \\
\hline \multicolumn{3}{|l|}{ Renda Familiar (salário mínimo regional)* } \\
\hline Menos de 01 salário & 09 & 6,1 \\
\hline 01 - 03 salários & 105 & 71,9 \\
\hline 03 - 05 salários & 20 & 13,7 \\
\hline Mais de 05 salários & 12 & 8,2 \\
\hline
\end{tabular}

Fonte: Banco de dados da pesquisa "O perfil do cliente oncológico de um ambulatório de radioterapia" *Salário mínimo regional no período de coleta dos dados, R\$ 378,00. 


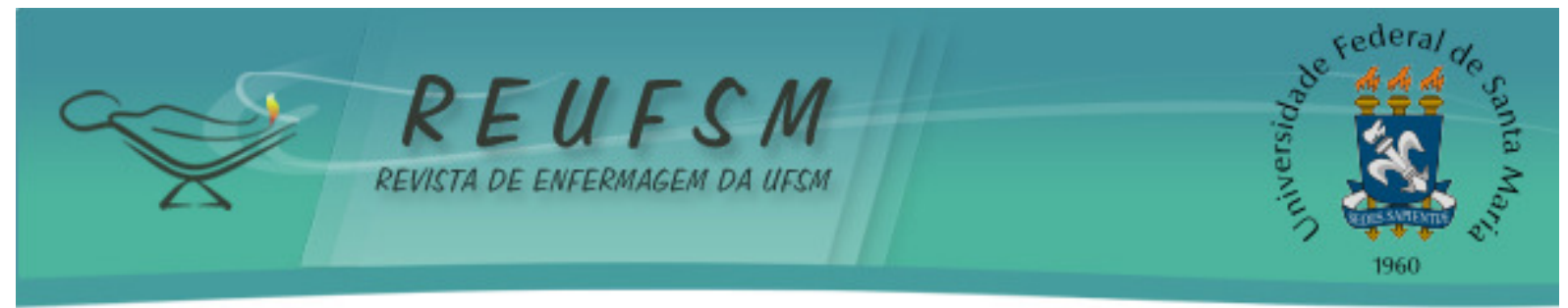

$\mathrm{Na}$ Tabela 2, estão apresentados os resultados encontrados para os fatores de risco, como o consumo de tabaco, de álcool e de chimarrão.

Tabela 2 - Distribuição dos fatores de risco dos clientes com câncer em tratamento radioterápico na Região Sul do Brasil, 2008

\begin{tabular}{lcc}
\hline \multicolumn{1}{c}{ Caracterização dos fatores de risco } & N (147) & (\%) \\
\hline Tabagismo & 105 & 71,4 \\
Sim & 42 & 28,6 \\
Não & & \\
Tempo de tabagismo* & 06 & 5,7 \\
$\quad$ Menos de 05 anos & 07 & 6,7 \\
$5-10$ anos & 13 & 12,4 \\
$10-20$ anos & 79 & 75,2 \\
Mais de 20 anos & & \\
Álcool & 103 & 70,1 \\
Sim & 44 & 29,9 \\
Não & & \\
Chimarrão & 03 & 2,0 \\
01 vez na semana & 10 & 6,8 \\
03 vezes na semana & 23 & 15,7 \\
Eventualmente & 75 & 51,0 \\
Todos os dias & 35 & 23,8 \\
$\quad$ Nunca ingeriu & & \\
\hline
\end{tabular}

Fonte: Banco de dados da pesquisa "O perfil do cliente oncológico de um ambulatório de radioterapia"

*Variável com o maior número de ignorados $(\mathrm{n}=105)$.

A Tabela 3 apresenta as neoplasias mais frequentes e o tratamento precedente ao radioterápico. 


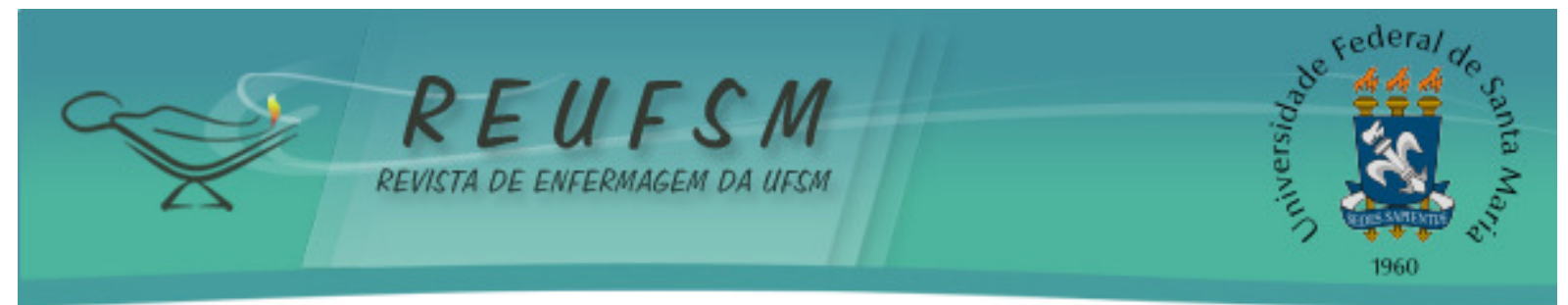

Tabela 3 - Distribuição dos clientes segundo tipo de câncer e tratamento anterior a radioterapia, na Região Sul do Brasil, 2008

\begin{tabular}{lcc}
\hline \multicolumn{1}{c}{ Características da doença } & N (147) & \% \\
\hline Tipo de câncer & & \\
Câncer de nasofaringe & 58 & 42,0 \\
Câncer de mama & 15 & 10,9 \\
Câncer de próstata & 14 & 10,1 \\
Câncer de pulmão & 12 & 8,7 \\
Câncer de bexiga & 05 & 3,6 \\
Outros tipos de câncer & 34 & 24,6 \\
Tratamento anterior à radioterapia & 70 & 47,6 \\
Cirúrgico & 56 & 38,1 \\
Quimioterápico & 05 & 3,4 \\
Hormonal & 04 & 2,7 \\
Radioterapia & 01 & 0,7 \\
Braquiterapia & & \\
\hline
\end{tabular}

Fonte: Banco de dados da pesquisa "O perfil do cliente oncológico de um ambulatório de radioterapia"

\section{DISCUSSÃO}

O câncer atinge todas as faixas etárias, independente de raça, sexo ou condições socioeconômicas, e representa a segunda causa de morte no Brasil. ${ }^{1}$ Com relação ao sexo, a prevalência de câncer entre homens e mulheres é muito similar nos países desenvolvidos, enquanto nos países em desenvolvimento, a prevalência nas mulheres é 25,0\% maior. Entretanto, os homens são acometidos pelas localizações de câncer com pior sobrevida, tais como fígado, esôfago e estômago. ${ }^{7}$

Um estudo utilizando dados do Sistema de Informação sobre Mortalidade do Ministério da Saúde, referente aos óbitos por neoplasia de indivíduos acima de 30 anos no período de 1980 a 2000 em 10 capitais do país, calculou o coeficiente de mortalidade segundo sexo, grupos etários e capitais em cada ano investigado. Os pesquisadores constataram que a mortalidade foi superior para o sexo masculino e indivíduos acima de 60 anos devido à maior prevalência de câncer letal entre os homens e ao aumento da expectativa de vida. A mortalidade por neoplasia vem aumentando e o câncer pode tornarse a principal causa de morte no Brasil. ${ }^{8}$

A partir das frequências reveladas na caracterização da amostra do estudo, observa-se uma população predominantemente idosa. 0 avançar da idade associado ao processo de envelhecimento eleva a probabilidade de o indivíduo desenvolver algum tipo de câncer concomitante a alguma outra condição crônica já instalada. Esta condição geralmente implica na necessidade de cuidados de saúde contínuos e especializados.

A situação conjugal pode ser uma importante fonte de apoio e de cuidado, na presença de uma afecção crônica degenerativa como o câncer. Desse modo, a ausência de companheiro e (ou) filho pode ser um fator a comprometer a qualidade de vida dos idosos, principalmente se vierem a perder a autonomia. ${ }^{9}$

Um dos fatores agravantes evidenciados foi o baixo nível de escolaridade. Em um estudo realizado com mulheres acometidas por câncer de mama, observou-se que houve maior sobrevida global em cinco anos no grupo de mulheres com maior escolaridade. ${ }^{10}$ 


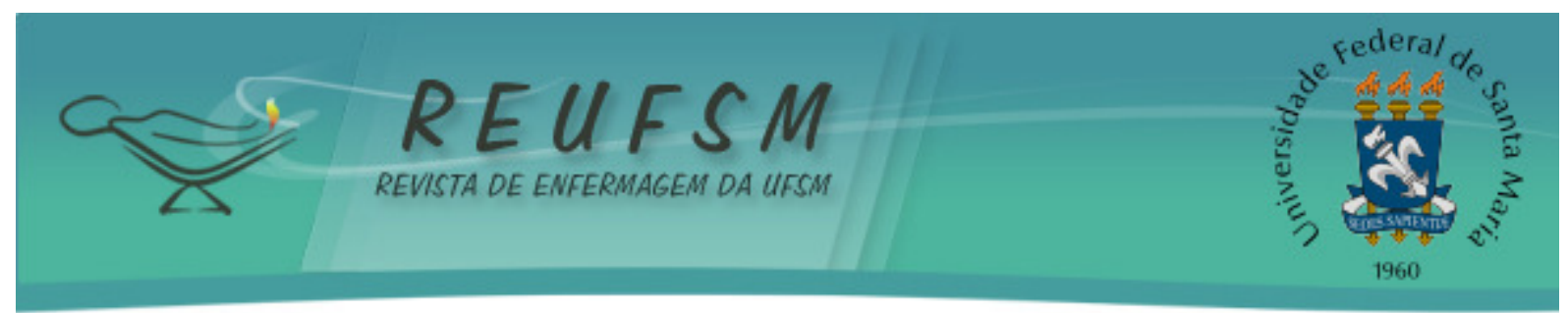

Além disso, a escolaridade tem influência no acesso aos serviços de saúde e interfere na capacidade de entendimento, na compreensão do tratamento prescrito, na realização do autocuidado e na relação terapêutica entre profissional de saúde e usuário. ${ }^{11-12}$ Dessa forma, a escolaridade deveria ser considerada como uma das diferentes ações vinculadas às estratégias de prevenção do câncer e promoção da saúde.

Evidencia-se o baixo poder aquisitivo entre os entrevistados, pois $71,9 \%$ relataram viver com renda familiar de até três salários mínimos regionais. A situação socioeconômica desempenha papel fundamental na determinação da saúde de indivíduos e populações. Assim, indivíduos com baixa renda apresentam uma pior condição de saúde e menor procura por serviços médicos e acesso aos cuidados de saúde. ${ }^{11-13}$ Ademais, as doenças crônicas não transmissíveis podem reduzir a capacidade para o trabalho das pessoas e resultar em maiores gastos com o tratamento, podendo aumentar as dificuldades financeiras. ${ }^{1}$

Um exemplo dado é o prognóstico do câncer de mama que está associado ao nível socioeconômico, pois, em classes sociais desfavorecidas, o diagnóstico pode ocorrer em uma fase mais avançada da doença em decorrência da dificuldade de acesso a ações de prevenção e cuidados de saúde. ${ }^{14}$ Neste sentido, há necessidade de intervenção, principalmente em mulheres com condições socioeconômicas limitadas de acesso à saúde e com idade mais avançada. ${ }^{15}$

Em um estudo foi evidenciado que as classes sociais economicamente desfavorecidas são menos assistidas no que diz respeito ao controle do câncer. Deste modo, essas pessoas são mais expostas aos fatores de risco e comorbidades, além de se depararem com a dificuldade de acesso aos serviços de saúde, repercutindo no retardo do diagnóstico, podendo muitas vezes levar a um prognóstico ruim, e consequentemente a intervenções mutiladoras. ${ }^{16}$

Em relação aos fatores de risco para o câncer, ocupam lugar de destaque o tabagismo e o consumo de álcool. ${ }^{1}$ Constatou-se no presente estudo elevada prevalência de clientes que fizeram uso dessas substâncias, sendo que, para o tabaco, o consumo foi superior a 20 anos. 0 tabagismo constitui a principal causa de morte evitável no mundo e é um dos importantes fatores de risco para doenças crônicas. A partir disso, os órgãos públicos vêm desenvolvendo estratégias de prevenção e de controle quanto ao seu uso, sendo exemplo o crescente número de campanhas veiculadas pela mídia sobre a proibição do uso do tabaco em locais públicos. ${ }^{17}$

O tabagismo contribui não somente para o aumento da incidência de câncer de pulmão, mas também para a elevação de casos novos de câncer de laringe, esôfago, boca e faringe. Nestes dois últimos tipos de câncer, a incidência se eleva se o tabagismo estiver associado ao consumo de álcool e a más condições nutricionais. ${ }^{1-7}$ Esta afirmativa vem ao encontro com os achados deste estudo que teve o câncer de nasofaringe como o mais frequente.

Existem outros hábitos que podem estar associados ao surgimento de câncer na cavidade oral, independentemente do consumo de álcool e tabaco. Dentre eles, estão a ausência de consultas com dentistas, o sangramento gengival prolongado e o uso irregular de enxaguatórios bucais. ${ }^{18}$

Além dos fatores de risco relacionados acima está o uso do chimarrão, hábito comum na região sul do Brasil, o que pode se caracterizar como um agravante para a ocorrência de câncer de esôfago. ${ }^{7} \mathrm{~A}$ água quente consumida no chimarrão pode ser um fator importante no surgimento do câncer esofágico. Fato evidenciado em um estudo realizado no município de Taquara/RS, em 36 residências, em que 107 pessoas estavam ingerindo chimarrão no momento da coleta de dados. ${ }^{19}$

De acordo com o Instituto Nacional do Câncer (INCA), o surgimento do câncer é multifatorial. Desse modo, álcool, tabaco, residir em zona rural, tem associação com o câncer de esôfago, enquanto álcool, tabaco, chimarrão, churrasco e preparo de alimentos 


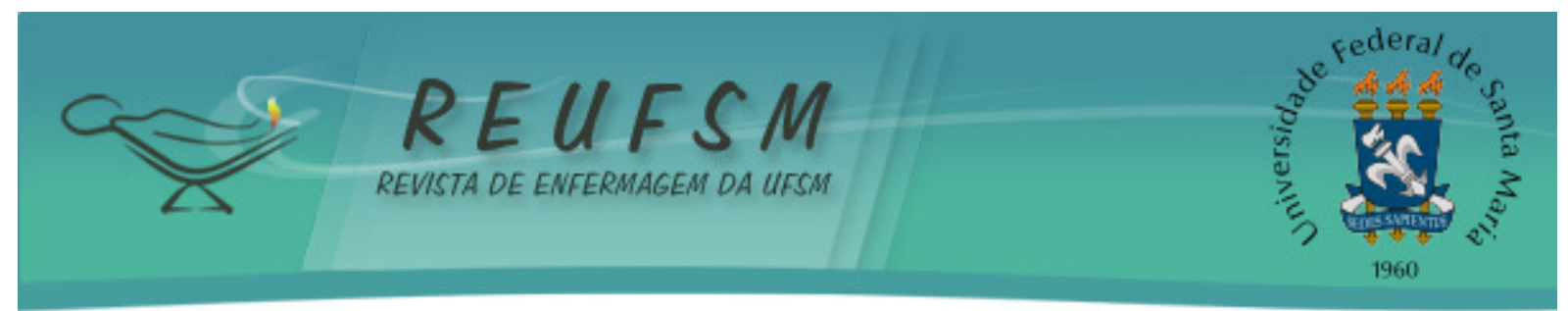

em fogão a lenha, estão relacionados com o câncer da cavidade bucal. ${ }^{20}$ Nesse contexto, destaca-se que este estudo foi realizado em uma região, em que costumes como o hábito de ingerir chimarrão e o churrasco são práticas tradicionais no sul do Brasil.

Quanto à modalidade de tratamento utilizada antes da radioterapia, a maioria dos clientes realizou cirurgia oncológica prévia. Dados da Organização Mundial da Saúde apontam que dois terços $(70,0 \%)$ dos clientes portadores de câncer utilizaram a radioterapia em algum momento do tratamento, quer de maneira isolada, quer associada a outras terapias oncológicas. ${ }^{21}$ A radioterapia isolada ou combinada com a cirurgia, com a quimioterapia ou com ambas é uma modalidade terapêutica efetiva para muitas neoplasias malignas e apresenta taxas de sobrevida altas no tratamento do câncer em estádios I e II. ${ }^{22}$

A radioterapia é uma terapêutica essencial para a cura ou remissão da doença, mas pode gerar situações inesperadas para os clientes e seus familiares que passam a requerer novas práticas de cuidado de modo a auxiliar no tratamento estabelecido. ${ }^{23}$

Tendo em vista o longo período terapêutico, riscos de complicações, sequelas e incapacidades funcionais significativas, o câncer acarreta a necessidade de um controle rigoroso e demanda o envolvimento da família no processo de cuidado do familiar acometido pela doença. ${ }^{24}$

A partir dos dados apresentados, sugere-se a realização de estudos que elucidem a associação das variáveis do perfil do cliente oncológico ao surgimento do câncer. Considera-se que este oferecerá subsídios sobre os componentes estruturais a fim de que o serviço de saúde possa criar um ambiente favorável para melhorar a adesão e o aperfeiçoamento das estratégias de prevenção, de controle e de tratamento das condições crônicas, especificamente o câncer, pela sua elevada morbidade e mortalidade.

Assim, conhecer as características das pessoas com câncer assistidas no serviço de radioterapia permite que o profissional da saúde possa desenvolver práticas assistenciais mais humanizadas e qualificadas a esta população. ${ }^{25}$

\section{CONCLUSÃO}

Este estudo visou contribuir para a construção de uma assistência de qualidade aos clientes com câncer em tratamento radioterápico, pois possibilita descrever as características sociodemográficas e as relacionadas ao adoecimento e ao tratamento. Conforme observado, a população do estudo foi predominantemente constituída pelo sexo masculino, na faixa etária de 60 anos ou mais, vivendo casados/com companheiros, apresentando reduzida renda familiar e baixa escolaridade. 0 câncer mais frequente foi o de nasofaringe, seguido pelo câncer de mama, próstata e pulmão.

Entende-se que, conhecendo o perfil desta clientela, podem-se desenvolver estratégias de fortalecimento de ações em saúde e a implementação de propostas de cuidado direcionadas à promoção e à prevenção do câncer juntamente com os usuários do serviço de saúde e seus familiares.

A partir disto, ressalta-se que a realização de estudos epidemiológicos têm sido de extrema importância para caracterizar os clientes com câncer e os fatores de risco possivelmente associados a essa doença. Dessa forma, este tipo de investigação continua sendo um dos melhores recursos de que se dispõe para direcionar ações preventivas e de detecção precoce.

Como limitação para a realização deste estudo, pode-se apontar o reduzido tamanho da amostra, o que está relacionado à queda de $50,0 \%$ dos atendimentos pelo Centro Regional de Oncologia durante a coleta de dados devido a reformas na área física do serviço. 


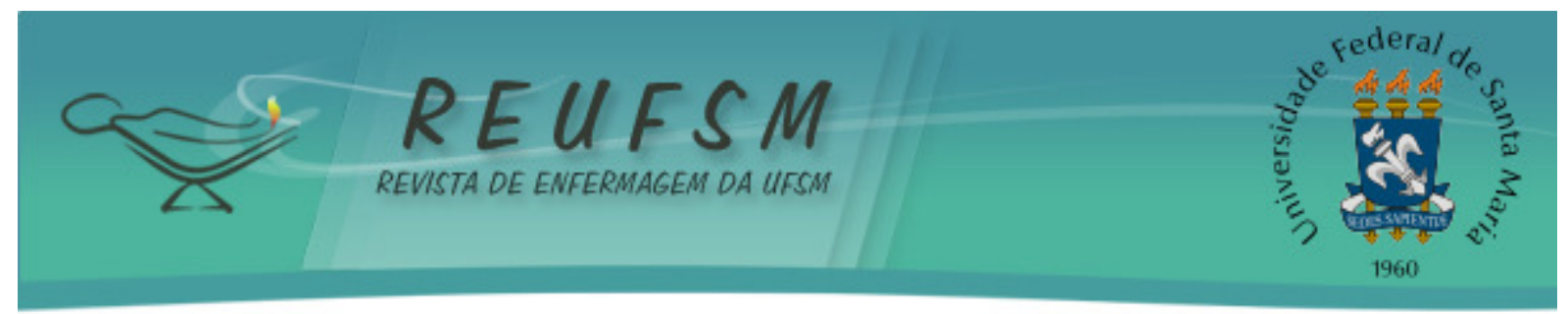

\section{REFERÊNCIAS}

1. Ministério da Saúde (BR). Instituto Nacional de Câncer José Alencar Gomes da Silva. Estimativa 2012: incidência de câncer no Brasil. Rio de Janeiro (RJ): INCA; 2011.

2. Otto SE. Oncologia. $1^{\text {a }}$ ed. Rio de Janeiro (RJ): Reichmann \& Affonso Editores; 2002.

3. Brasil. Lei $n^{\circ} 12.732$, de 22 de novembro de 2012. Dispõe sobre o primeiro tratamento de paciente com neoplasia maligna comprovada e estabelece prazo para seu início. Diário Oficial da União. Brasília, 23 nov. 2012; Seção 1:1-2.

4. Rodrigues JSM, Ferreira NMLA. Caracterização do perfil epidemiológico do câncer em uma cidade do interior paulista: conhecer para intervir. Rev Bras Cancerol. 2010;56(4):431-41.

5. Kraft Rovere R, Dagnoni C, Corrêa EG, Oliveira ED, Figueira FC, Sapelli J. Profile of cancer patients treated at the emergency room of a tertiary cancer care centre in southern Brazil. Klin Onkol. 2012;25(6):452-6.

6. Ministério da Saúde (BR). Conselho Nacional de Saúde. Resolução 196, de 10 de outubro de 1996: diretrizes e normas regulamentadoras de pesquisa envolvendo seres humanos. Brasília (DF); 1996.

7. Guerra MR, Gallo CVM, Mendonça GAS. Risco de câncer no Brasil: tendências e estudos epidemiológicos mais recentes. Rev Bras Cancerol [Internet]. 2005 maio [acesso em 2011 fev 27];3(51):227-34. Disponível em: http://www.eteavare.com.br/arquivos/81_392.pdf

8. Cervi A, Hermsdorff HHM, Ribeiro RCL. Tendência da mortalidade por doenças neoplásicas em 10 capitais brasileiras, de 1980 a 2000. Rev Bras Epidemiol [Internet]. 2005 set [acesso em 2010 jul 15];8(4):407-18. Disponível em: http://www.scielo.br/pdf/rbepid/v8n4/07.pdf

9. Caetano JA, Costa AC, Santos ZMSA, Soares E. Descrição dos fatores de risco para alterações cardiovasculares em um grupo de idosos. Texto \& Contexto Enferm. 2008;17(2):327-35.

10. Schneider IJC, D’Orsi E. Sobrevida em cinco anos e fatores prognósticos em mulheres com câncer de mama em Santa Catarina, Brasil. Cad Saúde Pública. 2009;25(6):1285-96.

11. Migowski A, Silva GA. Sobrevida e fatores prognósticos de pacientes com câncer de próstata clinicamente localizado. Rev Saúde Pública. 2010;44(2):344-52.

12. Meireles VC, Matsuda LM, Coimbra JAH, Mathias TAF. Características dos idosos em área de abrangência do Programa Saúde da Família na Região Noroeste do Paraná: contribuições para a gestão do cuidado em enfermagem. Saúde Soc. 2007;16(1):69-80.

13. Lima-Costa MF, Barreto SM. Tipos de estudos epidemiológicos: conceitos básicos e aplicações na área do envelhecimento. Epidemiol Serv Saúde. 2003;12(4):189-201.

14. Abreu E, Koifman S. Fatores prognósticos no câncer de mama feminino. Rev Bras Cancerol [Internet]. 2002 jan-mar [acesso em 2011 mar 2];48(1):113-31. Disponível em: http://www.inca.gov.br/rbc/n_48/v01/pdf/revisao.pdf.

15. Martins LFL, Valente JG, Thuler LCS. Factors related to inadequate cervical cancer screening in two Brazilian state capitals. Rev Saúde Pública. 2009;43(2):318-25.

16. Wünsch Filho V, Antunes JLF, Boing AF, Lorenzi RL. Perspectivas da investigação sobre determinantes sociais em câncer. Physis (Rio J). 2008;18(3):427-50. 


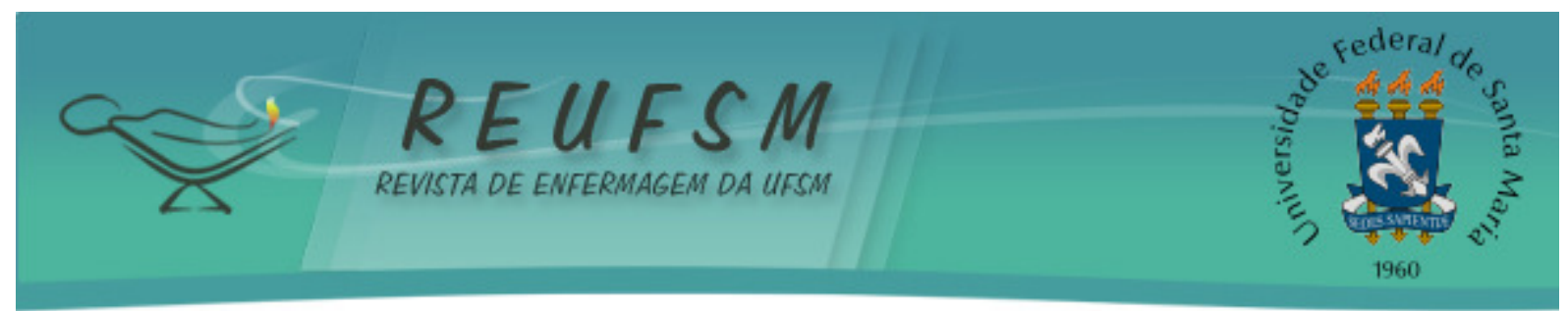

17. Hallal ALC, Gotlieb SLD, Almeida LM, Casado L. Prevalência e fatores associados ao tabagismo em escolares da Região Sul do Brasil. Rev Saúde Pública. 2009;43(5):779-88.

18. Marques LA, Eluf-Neto J, Figueiredo RAO, Góis-Filho JF, Kowalski LP, Carvalho MB, et al. Oral health, hygiene practices and oral câncer. Rev Saúde Pública. 2008;42(3):471-9.

19. Barros SGS, Ghisolfi ES, Luz LP, Barlem GG, Vidal RM, Wolff FH, et al. Mate (chimarrão) é consumido em alta temperatura por população sob risco para o carcinoma epidermóide de esôfago. Arq Gastroenterol. 2000;37(1):25-30.

20. Instituto Nacional do Câncer. Prevenção e fatores de risco [Internet]. [acesso em 2013 maio 8]. Disponível em: http://www.inca.gov.br/conteudo_view.asp?id=13.

21. World Health Organization (WHO). Fight against cancer: strategies that prevent, cure and care. Genebra: WHO; 2007.

22. Albuquerque ILS, Camargo TC. Prevenção e tratamento da mucosite oral induzida por radioterapia: revisão de literatura. Rev Bras Cancerol. 2007;53(2):195-209.

23. Muniz RM. Os significados da experiência da radioterapia oncológica na visão de pacientes e familiares cuidadores [tese]. Ribeirão Preto: Universidade de São Paulo, Escola de Enfermagem de Ribeirão Preto; 2008. 243 p.

24. Visoná F, Prevedello M, Souza EN. Câncer na família: percepções de familiares. Rev Enferm UFSM [Internet]. 2012 jan-abr [acesso em 2013 abr 5];2(1):145-55. Disponível em: http://cascavel.ufsm.br/revistas/ojs-2.2.2/index.php/reufsm/article/view/3943/3148

25. Santos AG, Costa JP, Barros IC, Almeida LHRB. Characterization of cancer pain in patients submitted to radiotherapy. Rev Enferm UFPE on line [Internet]. 2012 Sept [acesso em 2013 maio 8];6(9):2111-8. Disponível em: http: //www.revista.ufpe.br/revistaenfermagem/index.php/revista/article/viewArticle/2959

Data de recebimento: 08/04/2013

Data de aceite: $30 / 07 / 2013$

Contato com autor responsável: Eda Schwartz

E-mail: eschwartz@terra.com.br

Endereço: Avenida Fernando Osório, $\mathrm{n}^{\circ}$ 5189B - Bairro Três Vendas, Pelotas, RS, Brasil. CEP: $96065-000$ 